Running title: Branched-chain amino acid biosynthesis inhibitors

Corresponding author:

Mercedes Royuela

Departamento de Ciencias del Medio Natural

Universidad Pública de Navarra

Campus de Arrosadía, E-31006 Pamplona, Spain

Tel: +34 948169120

Fax: +34 948168930

E-mail: royuela@unavarra.es 


\section{Branched-chain amino acid biosynthesis inhibitors: herbicide efficacy is associated with an induced carbon- nitrogen imbalance}

Ana Zabalza, Amaia Zulet, Miriam Gil-Monreal, Maria Igal, Mercedes Royuela*

Departamento de Ciencias del Medio Natural, Universidad Pública de Navarra, Campus Arrosadia, E-31006, Pamplona, Spain

(C) 2013. This manuscript version is made available under the CC-BY-NC-ND 4.0 license http://creativecommons.org/licenses/by-nc-nd/4.0/ 


\section{Summary}

Acetolactate synthase (ALS; EC 4.1.3.18) and ketol-acid reductoisomerase (KARI; EC 1.1.1.86) are two consecutive enzymes in the biosynthesis of branched-chain amino acids. Several commercial herbicides inhibit ALS as their primary site of action. KARI has also attracted attention as a potential target for herbicides. Although potent and selective inhibitors of KARI have been discovered, these inhibitors display less herbicidal activity than ALS-inhibiting herbicides. To obtain a better understanding of these findings, we have compared the physiological effects induced in pea plants after KARI or ALS inhibition. Although, both types of inhibitors induce growth arrest and photosynthesis inhibition, plant death occurs more rapidly under ALS inhibition than KARI inhibition. Carbohydrates accumulated in the leaves and roots following treatments with both inhibitors. The carbohydrate accumulation in the leaves occurred as a consequence of a decrease in sink strength. In contrast, the free amino acid content was only affected through ALS inhibition. These results indicate that although KARI and ALS inhibition block the same biosynthetic pathway and exert common effects on carbon metabolism, nitrogen metabolism is more affected via ALS than KARI inhibition. Thus, metabolic alterations in nitrogen metabolism induced through ALS inhibitors might contribute to the increased efficacy of these chemicals as herbicides.

Keywords: mode of action; herbicide; branched-chain amino acid; acetolactate synthase; ketol-acid reductoisomerase.

Abbreviations: ALS, acetolactate synthase; BCAA, branched-chain amino acid; CPCA, cyclopropane-1,1-dicaboxylic acid; KARI, ketol-acid reductoisomerase; IM, imazethapyr. 


\section{Introduction}

Valine, leucine and isoleucine form the small group of branched-chain amino acids (BCAAs). Bacteria, archaea, fungi and plants synthesise BCAAs, while animals, including humans, are not able to synthesise BCAAs de novo and have to acquire these amino acids through their diets.

A unique feature of BCAA biosynthesis is that valine and isoleucine are synthesised in two parallel pathways through a single set of four enzymes, acetolactate synthase or acetohydroxy acid synthase (ALS; EC 4.1.3.18), ketol-acid reductoisomerase or acetohydroxy acid isomeroreductase (KARI; EC 1.1.1.86), dihydroxy-acid dehydratase and branched-chain aminotransferase, which catalyse the formation of these two amino acids using different substrates. ALS catalyses the condensation of either two molecules of pyruvate to form acetolactate or one molecule of pyruvate and one molecule of 2-ketobutyrate to form 2-aceto-2hydroxybutyrate (Singh, 1999). KARI catalyses the reductive isomerisation of AL to 2,3-dihydroxy3-isovalerate or the conversion of 2-aceto-2-hydroxybutyrate to 2,3-dihydroxy-3-methylvalerate (Durner et al., 1993).

ALS is the best-studied enzyme involved in BCAA metabolism because it is the target for commercially successful herbicides. There are five different chemical classes of herbicides that inhibit ALS: sulfonylureas, imidazolinones, triazolopyrimidines, sulfonylaminocarbonyl triazolinones and pyrimidinyl-oxy-benzoates. These chemicals have emerged since the 1980s and have been demonstrated as potent, selective, broad-spectrum herbicides and inhibitors of plant growth. Due to their high efficacy, these inhibitors are used at low field application rates in the range of grams per hectare, while other herbicides are applied in the range of kilograms per hectare. The efficacy and potency of ALS inhibitors have ensured the continued success of these herbicides, which have rapidly challenged, and in some instances replaced, traditional products, particularly in cereals and soybeans (Cobb and Reade, 2010). Currently, ALS inhibitors represent the second largest class of active herbicidal products and are traditionally used in weed control for many non-transgenic crops. The combination of the widespread usage of ALS-inhibiting herbicides and the development of resistance to these herbicides has resulted in the evolution of 127 ALS inhibitor-resistant weed 
species (Heap, 2012). In most cases, where the underlying resistance mechanism has been investigated, resistance occurs through point mutation(s) in the ALS gene that reduce the sensitivity of the enzyme to herbicides (Powles and Yu, 2010). The characteristic attributes of ALS inhibitors (i.e., their low toxicity and high efficacy) indicate that the inhibition of BCAA biosynthesis is a suitable target for herbicidal action; however, the rapid evolution of weed resistance to ALS inhibitors limits the applicability of these compounds and represents a significant challenge. New chemistry and novel herbicides with unique modes of action are needed to manage the evolution of resistance of weeds to existing herbicides (Gewick, 2010; Duke, 2012).

A major research effort has emerged for the development of new products to inhibit the synthesis of branched-chain amino acids through the inhibition of another enzyme in the same pathway, thereby targeting inhibitors that might behave as herbicides, similar to ALS inhibitors, but function via a new mode of action, thereby avoiding the selection pressure on ALS. Thus, efficient inhibitors of all the enzymes in the branched-chain amino acid pathway, with similar in vitro Ki values have been identified, but only inhibitors of ALS have been commercialised (Wittenbach and Abell, 1999; Duke, 2012). Compounds such as 2-dimethylphosphinoyl-2-hydroxyacetic acid (Hoe 704) and N-hydroxy-N-isopropyloxamate (IpOHA) are potent and selective competitive inhibitors of KARI (Schulz et al., 1988; Aulabaugh and Schloss, 1990) but only display minor herbicidal activity. Two explanations have been suggested for their lack of potency in the field: these compounds exhibit slow binding inhibition of KARI in solution and act as competitive inhibitors that prevent the optimal effects of the inhibitor through the accumulation of substrates (Leung and Guddat, 2009). Cyclopropane-1,1-dicaboxylic acid (CPCA) acts as a KARI inhibitor in Escherichia coli in vitro and in some weeds in vivo (leaf disks) (Gerwick et al., 1993). This inhibition has been enzymatically characterised in rice, showing that CPCA mimics the transition state of the reaction. Nevertheless, this inhibition is substantially less potent in vivo than suggested by the effects on KARI in vitro (Lee et al., 2005), even when different substitutions are tested (Liu et al., 2007; 2011).

After an inhibitor reaches its primary target, several physiological effects are triggered within the plant. Although the biochemical mechanism underlying the blocking of ALS activity 
through ALS inhibitors has been extensively studied, little is known regarding the physiological process underlying plant death resulting from inactivated ALS.

In general, after the target of an inhibitor has been affected, death occurs as a result of various causes. First, death could be associated with the lack of end products generated from the inhibited pathway. Second, plant death could result from an accumulation or increased availability of the substrates of the inhibited enzymatic pathway. Third, lethality could be associated with several side reactions triggered after the inhibition of the target. Thus, the differences in herbicidal efficacy detected between ALS and KARI inhibitors might reflect the physiological processes leading to plant death. The two types of inhibitors should have similar effects if lethality results from the lack of end products (i.e., valine, leucine and isoleucine), but these compounds would be expected to perform differently if plant death is more associated with substrate accumulation or side effects.

An impairment of carbon and nitrogen metabolism has been reported in treated plants following ALS inhibition (Zabalza et al., 2004; 2005; 2006). In contrast, although several aspects of the physiological response after KARI inhibition have been reported (Wittenbach and Abell, 1999), no exhaustive studies on the effects of these inhibitors on carbon or nitrogen metabolism have been performed.

The aim of this study was to characterise the effects of a KARI inhibitor on carbon and nitrogen metabolism compared with an ALS inhibitor. To this end, pea plants were treated with imazethapyr (IM, an ALS inhibitor) or CPCA (a KARI inhibitor), and the effects of these compounds on carbohydrate and amino acid contents were compared, as these metabolic indicators are altered following ALS inhibition. CPCA was supplied at two different concentrations to determine whether the physiological responses were dose dependent. 


\section{Materials and methods}

\section{Plant material and treatment application.}

Pisum sativum L. cv. Snap Sugar Boys were grown in an aerated hydroponic culture in a growth chamber (Zabalza et al., 2005). When the plants were 12 days old, the tanks were divided into four groups: one control group and three inhibitor-treated groups. The ALS-inhibiting herbicide IM (commercial formula, Pursuit 10, BASF Española SA Barcelona, Spain) was applied to the nutrient solution at a concentration of $69 \mu M$ (20 mg active ingredient $\left.\mathrm{L}^{-1}\right)$. CPCA (1,1cyclopropanedicarboxylic acid), a KARI inhibitor, was applied to the nutrient solution at concentrations of 200 or $500 \mu \mathrm{M}$ (26 and $65 \mathrm{mg} \mathrm{L}^{-1}$, respectively). The nutrient solution was replaced every 3 days to maintain constant inhibitor concentrations in the nutrient solution throughout the experiment. The experiments were repeated in two independent series, with three replicate tanks per treatment.

The pea growth was determined using root and shoot lengths as the best indicators of growth inhibition. For metabolite analyses, leaf and root samples were collected at $0,1,3,7,10$ and 15 days after treatment. Plants were also harvested at 22 days after both CPCA treatments. At harvest, leaf and root samples were collected, immediately frozen in liquid nitrogen and stored at $-80^{\circ} \mathrm{C}$ until further analysis.

\section{Gas exchange measurements}

The net $\mathrm{CO}_{2}$ assimilation rate was measured in the youngest fully expanded leaf of intact plants using the portable ADC-LCi Infrared Gas Analyzer System (ADC BioScientific Ltd., Herts England). The leaf area was determined using the Li-3000 system (Li-Cor, Lincoln, Nebraska, USA).

\section{Determination of metabolite contents}

Acetolactate was extracted from the leaves and roots and analysed as previously described (Zabalza et al., 2005). The extraction and quantification of quinate through ion chromatography and amino acids via capillary electrophoresis was performed according to Orcaray et al. (2010). The glucose, fructose and sucrose contents were determined in ethanol-soluble extracts, and the ethanol-insoluble residue was extracted for starch analysis. The concentrations of starch and 
soluble sugars were determined using capillary electrophoresis according to the methods of Zabalza et al. (2004).

\section{Statistical analysis}

The mean values were calculated using the samples obtained from individual plants as replicates. The results were subjected to a separate one-way ANOVA for each day of treatment SPSS 16.0). The means were separated using the least significant difference method $(p<0.05$, Fisher protected). Significant differences between each treatment and the control plants (nottreated plants) are highlighted in the figures using a different symbol for each treatment. When the obtained values were percentages, a prior transformation to arcsine $\sqrt{ }(x / 100)$ was applied. 


\section{Results}

The addition of $69 \mu \mathrm{M}$ IM to the nutrient solution produced root and shoot growth inhibition (Fig. 1). The effects detected after IM treatment were used as references to determine the concentration of the other inhibitors, and preliminary analyses were conducted to determine the CPCA concentrations that induce similar effects (in terms of speed and intensity). Two CPCA concentrations were adopted (200 and $500 \mu \mathrm{M})$ to compare the effects obtained following IM treatment. Although root growth was arrested and shoot growth was suppressed after the administration of CPCA to the nutrient solution (Fig. 1), the pea growth was more severely inhibited after IM treatment. The administration of 200 and $500 \mu \mathrm{M}$ CPCA arrested root growth on the 7th and 10th day after the onset of treatment, respectively, and the inhibition of shoot growth was observed from day 10 of the treatment period.

Plant death occurred at 22 days after IM treatment (net photosynthesis, almost null). The death of pea plants following KARI inhibition took longer than after ALS inhibition and occurred in a dose-dependent manner; 200 and $500 \mu \mathrm{M}$ CPCA caused plant death after 36 and 31 days of treatment, respectively.

The addition of CPCA to the nutrient solution dramatically increased the acetolactate (the substrate of KARI) content in the leaves and roots from the beginning of the treatment (Fig. 2). This result confirms the effectiveness of $\mathrm{CPCA}$ as a KARI inhibitor in vivo. The acetolactate accumulation in the roots increased over a period of 10 days and was maintained thereafter (Fig. 2B). Although CPCA was absorbed in the roots, the acetolactate accumulation was higher in the leaves, with maximum accumulation detected after 3 days of treatment (Fig. 2A).

Although ALS inhibition reduced the net photosynthesis by day 7 , plants treated with 200 $\mu \mathrm{M}$ CPCA only showed an inhibition of photosynthesis at the end of the study (15-22 days), while plants treated with $500 \mu \mathrm{M} \mathrm{CPCA}$ were inhibited after the tenth day of treatment (Fig. 3). At the end of treatment, the leaves of plants treated with both CPCA doses showed low photosynthetic rates.

To the best of our knowledge, this study is the first to describe the effects of KARI inhibition on net photosynthesis (Fig. 3) and carbohydrate contents (Fig. 4 and 5). In general, all of the applied treatments resulted in carbohydrate accumulation in the leaves in the form of both soluble 
carbohydrates (fructose, glucose and sucrose) and starch from the onset of treatment, although several differences were detected. Under IM treatment, the metabolite content was only monitored until day 15 , and at day 22 , the plants were dead. IM treatment caused a greater increase in the fructose and glucose contents than that observed under CPCA treatment, whereas the sucrose accumulation was higher after KARI inhibition than after ALS inhibition. The glucose accumulation observed following CPCA treatment was dose dependent, with greater and earlier accumulation occurring in plants treated with $500 \mu \mathrm{M}$ CPCA than in those treated with $200 \mu \mathrm{M} \mathrm{CPCA}$ (Fig. 4B). The pattern of sucrose and starch accumulation was similar for both concentrations of CPCA tested (Fig. 4C and D). In both cases, the accumulation was significant after 3 days of treatment. Although the sucrose and starch accumulation was maintained for the duration of the experimental period in the leaves of pea plants supplied with $200 \mu \mathrm{M} \mathrm{CPCA}$, the leaves of pea plants treated with the highest concentration of CPCA showed sucrose and starch contents that were similar to those observed in the control leaves after 22 days.

Despite the accumulation of carbohydrates in the leaves, there was no carbohydrate shortage in the roots. Indeed, carbohydrates (both sucrose and starch) also accumulated in the roots when CPCA or IM was added to the nutrient solution. Similar to the leaves, the sucrose accumulation in the roots was dependent on the CPCA dose applied. The higher CPCA concentration $(500 \mu \mathrm{M})$ provoked a stronger effect on the sucrose content (Fig. 5A); however, the starch accumulation was not as great. The roots of pea plants treated with IM showed the highest starch accumulation on day 7 , and the values were close to the control values at days 10 and 15 . In CPCA-treated plants, the starch accumulation was only significant at 7, 10 and 15 days from the onset of treatment (Fig. 5B).

Fig. 6 shows the free amino acid contents and patterns of branched-chain and aromatic amino acids in the roots and leaves. Because these herbicide treatments induce carbohydrate accumulation in leaves and roots (Fig. 4 and 5), the content of the total free amino acid pool was expressed per gram of sugar-free fresh weight. Only the ALS inhibitor induced the short-term accumulation of the total free amino acid pool in both leaves and roots. The administration of CPCA to the nutrient solution did not change the short-term content of total amino acids in the 
leaves, but the amino acid content was reduced from day 15 of the study (Fig. 6A). The only modification observed in the roots was detected at 7 days after treatment, when both concentrations of CPCA caused a significant transient accumulation of free amino acids (Fig. 6D).

These results must be considered together to interpret the observed amino acid contents following these treatments, as a general increase in the total free amino acid content through proteolysis could ultimately mask the inhibitor-induced reduction in the synthesis of specific amino acids. Consequently, it is useful to express the specific amino acid content as a percentage of the total free amino acids, instead of an absolute value. The percentages of inhibited amino acid (branched chain) biosynthesis are provided (Fig. 6B and E). The percentage of branched-chain amino acids was increased in the leaves 7 days after IM and CPCA treatment (Fig. 6B). In the roots, after a transient reduction at 3 and 7 days, the percentage of branched-chain amino acids increased at the end of the study (Fig. 6E).

The well-known herbicide glyphosate inhibits aromatic amino acid biosynthesis. Recent studies have shown that ALS inhibitors and glyphosate share several physiological aspects in their modes of action (Orcaray et al., 2010; 2012), suggesting that there is a cross-regulation between the two biosynthetic pathways. Thus, it would be interesting to compare the effects on the percentage of aromatic amino acid content after the inhibition of branched-chain amino acid biosynthesis at different enzymatic points in the pathway. Fig. $6 \mathrm{C}$ and $\mathrm{F}$ show that the aromatic amino acid content was increased after the inhibition of ALS and KARI in both the leaves and roots.

The effect of the herbicides on the content of quinate, a compound synthesised in a lateral branch of the shikimate pathway, was also monitored for seven days (Fig. 7). Only the addition of the ALS inhibitor induced a significant increase in the quinate content of the leaves of treated plants after 3 days. Although after 7 days of treatment, the quinate accumulation was significant in plants treated with CPCA or IM, this accumulation was more prominent after ALS inhibition than after KARI inhibition. 


\section{Discussion}

Because ALS inhibition was identified as the target of a number of successful herbicides, the search for new herbicides targeting other enzymes involved in branched-chain amino acid biosynthesis has emerged. These studies have produced inhibitors for almost every step in the pathway. However, the amounts of these inhibitors required to control weeds are significantly higher compared with ALS inhibitors, thereby diminishing their usefulness as commercial herbicides. In this study, the effects of the inhibition of KARI, the second common enzyme in the biosynthetic pathway, on carbon and nitrogen metabolism were compared with the effects induced following ALS inhibition. The observed differences could potentially reflect differences in the herbicide efficacy between KARI and ALS inhibitors.

Although legume crops are tolerant to imazethapyr, the concentration of IM used in this study (20 mg active ingredient $\mathrm{L}^{-1}$ ) correspond to 100 times the recommended field application rate and was high enough to elicit ALS inhibition (45\% inhibition after 1 day and near $100 \%$ after 3 days) (Gaston et al., 2002). Furthermore, this treatment generates physiological effects similar to another ALS inhibitor, chlorsulfuron, to which the pea plant is susceptible (Zabalza et al., 2004; 2005; Orcaray et al., 2010; 2011).

The accumulation of acetolactate observed in this study confirmed that CPCA inhibited KARI, similar to the accumulation described in maize after Hoe 704 treatment (Schulz et al., 1988) and in Abutilon theophrasti after CPCA treatment (Gerwick et al., 1993). Both inhibitors caused plant death, growth inhibition and photosynthetic decline (Fig. 1, 2 and 3). Nevertheless, the three physiological effects occurred early under ALS inhibition than with KARI inhibition, in a dosedependent manner.

While carbohydrate accumulation in the leaves and roots has been described as a general physiological effect of ALS inhibition (Zabalza et al., 2004), this study is the first to demonstrate that starch and soluble carbohydrates accumulated in the leaves (Fig. 4) and roots (Fig. 5) following CPCA treatment. The simultaneous analysis of the carbohydrate contents facilitated the evaluation of the effect on phloem transport. As proposed for ALS inhibitors, an increase in the sucrose and starch contents in sinks suggests that sucrose is transported from the leaves to the 
roots at a higher rate than utilised in the sinks. Under these conditions, the sugar gradient required for long-distance transport is abolished; thus, phloem transport is inhibited, suggesting that the carbohydrate accumulation in the leaves of treated plants reflects a reduction in sink strength. The inhibition of carbon fixation was detected at 10 days after CPCA treatment (Fig. 3). As photosynthesis did not decline in the short term, it is reasonable to assume that the treated plants maintained sufficient photosynthetic carbon assimilation rates to accumulate carbohydrates in both sources and in sinks (Zabalza et al., 2004).

KARI inhibition blocked plant growth, but this inhibition was not due to a lack of respiratory substrates, as carbohydrates accumulated in both sources and in sink organs. The growth arrest detected after the inhibition of amino acid biosynthesis suggests that metabolism was impaired, which does not facilitate the utilisation of the available carbohydrates at the expected rate.

The net photosynthesis was reduced, and carbohydrates were accumulated in both the roots and leaves following ALS and KARI inhibition. Thus, it is reasonable to conclude that the modes of action of ALS and KARI inhibitors share these aspects. Moreover, other changes in carbon metabolism, such as the induction of ethanolic fermentation (Zabalza et al., 2005) and alternative oxidase (Gaston et al., 2003; Aubert et al., 1997), have been reported after both treatments. Taken together, these data suggest that plants treated with ALS or KARI inhibitors show a similarly altered carbon metabolism, in which the available carbohydrates are not consumed, and less efficient metabolic pathways are activated.

Similar ratios of branched-chain and aromatic amino acids were observed after KARI and ALS inhibition. The increase in the percentage of aromatic amino acids after ALS inhibition (Fig. 6C and F) has been previously described (Orcaray et al., 2010).

IM induced an increase in the free amino acid pool (Fig. 6), which is a well-known physiological effect of ALS inhibitors (Shaner and Raider, 1986; Anderson and Hibberd, 1985; Zabalza et al., 2006; Orcaray et al., 2010; Zabalza et al., 2011). Rhodes et al. (1987) proposed that this increased pool reflects an increase in protein turnover as a result of increased degradation and reduced synthesis rates. Indeed, although protein synthesis occurs after ALS inhibitor treatment, the amino-acid components of these proteins are typically not generated from newly incorporated 
nitrogen (Zabalza et al., 2006), but are primarily scavenged through protein degradation. Similarly to ALS inhibitors, other herbicides that inhibit amino acid biosynthesis have been reported to increase the free amino acid pool. Amino acid accumulation has been shown in pea plants treated with lethal doses of glyphosate (Orcaray et al., 2010; 2012), and glufosinate, an inhibitor of glutamine synthase, increases protein degradation (to release free amino acids) (Cobb and Reade, 2010). These data suggest the important role of this physiological effect on the lethality caused by these herbicides.

Interestingly, the effects of ALS and KARI inhibitors on the amino acid content were different, as no significant changes were detected after KARI inhibition (Fig. 6A and D), suggesting that ALS inhibition induces a greater alteration of nitrogen metabolism than KARI inhibition. As free amino acid accumulation has been reported after treatment with either of the three types of amino acid biosynthesis-inhibiting herbicides (ALS inhibitors, glyphosate and glufosinate), it is reasonable to proposed that this physiological effect is a marker of herbicide efficacy.

Quinate is a compound synthesised in a lateral branch of the shikimate pathway, occurring in relatively high concentrations in several types of tissues (Yoshida, 1975; Osipov and Aleksandrova, 1982; Albertini et al., 2006). Although its physiological role has not been completely clarified, quinate is considered as a reserve compound of the shikimate pathway. ALS inhibitors induce quinate accumulation in plant leaves (Orcaray et al., 2010). Moreover, exogenous quinate application had phytotoxic effects, showing that this plant metabolite induces the toxic effects of the herbicides; thereby, suggesting the importance of this metabolite in the mode of action of ALS inhibitors (Orcaray et al., 2010). Quinate accumulation after KARI inhibition was detected later, and this accumulation was not as prominent as observed with ALS (Fig. 7). Thus, similarly to growth inhibition and photosynthetic decline, these physiological effects occurred earlier under ALS than under KARI inhibition.

In this study, we used two different doses of the KARI inhibitor CPCA. Plants treated with the highest dose $(500 \mu \mathrm{M})$ of CPCA died, and several changes were more marked (rapid) than those produced in plants treated with a $200-\mu \mathrm{M}$ dose. These changes include an earlier reduction in the net photosynthesis, the increased accumulation of acetolactate in the leaves and roots and 
an increased glucose and sucrose accumulation in the leaves and roots, respectively, leading to the increased inhibition of plant growth. The metabolic changes induced through CPCA occurred in a dose-dependent manner. Furthermore, previous studies on whole plants or in cell culture have shown that the accumulation of acetolactate and acetoin (the product of the decarboxylation of acetolactate) is correlated with the phytotoxicity (Wittenbach et al., 1991), suggesting that these metabolic disorders are associated with lethality. Thus, plants treated with the highest inhibitor dose showed a more altered metabolism and died earlier than plants treated with the lowest dose.

Although the response of plants treated with KARI inhibitors is similar to that described for ALS inhibitors (Wittenbach and Abell, 1999), the results presented in this study showed that several important differences can be detected. The two types of inhibitors show common effects on carbon metabolism, but only the ALS inhibitor induced an increase in the free amino acid content. Thus, the imbalance in the carbon/nitrogen metabolism induced after ALS inhibition is more phytotoxic than that induced after KARI inhibition, reflecting the differences in herbicidal efficacy.

Studies on KARI and ALS inhibitors show that these two adjacent enzymes in the same pathway are not necessarily equal targets for herbicides. Several biochemical explanations have been proposed for the differences in herbicidal activity. First, the time required to achieve substantial inhibition through the application of IpOHA or Hoe704 in vitro is extremely long, which would considerably increase the time required for the inhibition of the target enzyme in vivo (Dumas et al., 1994). Second, a large excess of KARI in plants might disturb the effect of the inhibitors at the active site of the enzyme. Indeed, in barley, the level of KARI is 10 to 20 -fold higher than the level of ALS (Durner et al., 1993). Third, ALS undergoes irreversible inhibition in vivo in response to inhibitor binding (Hawkes and Thomas, 1990; Durner et al., 1991), while KARI inhibitors do not show this irreversibility (Durner et al., 1993; Aulabaugh and Schloss, 1990). Moreover, the results presented in this study suggest that the increased metabolic alterations induced through ALS inhibitors contribute to the enhanced performance of these compounds as commercial herbicides. 


\section{Acknowledgements}

The authors would like to thank Gustavo Garijo for technical assistance. M. Igal received a grant from the Public University of Navarre. A. Zulet and M. Gil-Monreal receive funding through fellowships from the Spanish Ministry of Education and the Public University of Navarre, respectively. This work was financially supported through grants from the Spanish Ministry of Education and Science (AGL-2010-18621).

\section{References}

Albertini MV, Carcouet E, Pailly O, Gambotti C, Luro F, Berti L. Changes in organic acids and sugars during early stages of development of acidic and acidless citrus fruit. J Agric Food Chem 2006; 54:8335-9.

Anderson PC, Hibberd KA. Evidence for the interaction of an imidazolinone herbicide with leucine, valine, and isoleucine metabolism. Weed Sci 1985;33:479-83.

Aubert S, Bligny R, Day DA, Whelan J, Douce R. Induction of alternative oxidase synthesis by herbicides inhibiting branched-chain amino acid synthesis. Plant J 1996;11:649-57.

Aulabaugh A, Schloss JV. Oxalyl hydroxamates as reaction-intermediate analogues for ketol-acid reductoisomerase. Biochem 1990;29:2824-30.

Cobb $\mathrm{AH}$, Reade JPH. The inhibition of amino acid biosynthesis. In: Herbicides and Plant Physiology, Ed. A.H Cobb; J.P.H. Reade. Oxford, UK: Wiley-Blackwell, 2010. p 176-199.

Duke SO. Why have no new herbicide modes of action appeared in recent years?. Pest Manag Sci 2012;68:505-12.

Dumas R, Cornillon-Bertrand C, Guigue-Talet P, Genix P, Douce R, Job D. Interactions of plant acetohydroxy acid isomeroreductase with reaction intermediate analogues: correlation of the slow, competitive, inhibition kinetics of enzyme activity and herbicidal effects. Biochem J 1994;301:813-20.

Durner J, Knorzer O, Böger P. Ketol-acid reductoisomerase from barley (Hordeum vulgare). Plant Physiol 1993;103:903-10. 
Durner J, Valérie Gailus V, Böger P. New aspects on inhibition of plant acetolactate synthase by chlorsulfuron and imazaquin. Plant Physiol 1991;95:1144-9.

Gaston S, Ribas-Carbo M, Busquets S, Berry JA, Zabalza A, Royuela M. Changes in mitochondrial electron partitioning in response to herbicides inhibiting branched-chain amino acid biosynthesis in soybean. Plant Physiol 2003;133:1351-9.

Gaston S, Zabalza A, Gonzalez EM, Arrese-Igor C, Aparicio-Tejo PM, Royuela M. Imazethapyr, an inhibitor of the branched-chain amino acid biosynthesis, induces aerobic fermentation in pea plants. Physiol Plant 2002;114:524-32.

Gerwick BC, Mireless LC, Eilers RJ. Rapid diagnosis of ALS/AHAS-resistant weeds. Weed Technol 1993;7:519-24.

Gerwick BC. Thirty years of herbicide discovery: surveying the past and contemplating the future. Agrow 2010; 600: vii-ix.

Hawkes TR, Thomas SE. Imidazolinones: Factors determining their herbicidal efficacy. In Barak Z, Chipman DM and Schloss JV, editors. Biosynthesis of branched chain amino acids, New York, USA: VCH Publishers. 1990. p. 373-89.

Lee YT, Ta HT, Duggleby RG. Cyclopropane-1,1-dicarboxylate is a slow-, tight-binding inhibitor of rice ketol-acid reductoisomerase. Plant Sci 2005;168:1035-40.

Leung EWW, Guddat LW. Conformational changes in a plant ketol acid reductoisomerase upon $\mathrm{Mg}^{2+}$ and NADPH binding as revealed by two crystal structures. J Mol Biol 2009;89:167182.

Liu XH, Chen PQ, Wang BL, Li YH, Wang SH, Li ZM. Synthesis, bioactivity, theoretical and molecular docking study of 1-cyano-N-substituted-cyclopropanecarboxamide as ketol-acid reductoisomerase inhibitor. Bioorg Medic Chem Let 2007;17:3784-8

Liu XH, Pan L, Tan CX, Weng JQ, Wang BL, Li ZM. Synthesis, crystal structure, bioactivity and DFT calculation of new oxime ester derivatives containing cyclopropane moiety. Pest Biochem Physiol 2011;101;143-7. 
Orcaray L, Igal M, Marino D, Zabalza A, Royuela M. The possible role of quinate in the mode of action of glyphosate and acetolactate synthase inhibitors. Pest Manag Sci 2010;66:262-9.

Orcaray L, Igal M, Zabalza A, Royuela M. 2011 Role of exogenously supplied ferulic and pcoumaric acids in mimicking the mode of action of acetolactate synthase inhibiting herbicides. J Agric Food Chem 2011;59:10162-68.

Osipov VI, Aleksandrova LP. Spatial organization of quinic and shikimic acid biosynthesis in autotrophic cells of Pinus sylvestris needles. Soviet Plant Physiol 1982;29:286-292.1982.

Powles SB, Yu Q. Evolution in action: plants resistant to herbicides. Annu Rev Plant Biol 2010; $61: 317-47$

Ray TB. The mode of action of chlorsulfuron: A new herbicide for cereals. Pest Biochem Physiol, $1982 ; 17: 10-17$

Rhodes D, Hogan AL, Deal L, Jamieson GC, Haworth P. Amino-acid metabolism of Lemna minor L .2. Responses to chlorsulfuron. Plant Physiol, 1987;84:775-80.

Schulz A, Sponemann P, Kocher H, Wegenmayer F. The herbicidally active experimental compound Hoe 704 is a potent inhibitor of the enzyme acetolactate reductoisomerase. FEBS Letters, 1988;238:375-8.

Shaner DL, Anderson PC, Stidham MA. Imidazolinones: Potent inhibitors of acetohydroxyacid synthase. Plant Physiol 1984;76:454-546.

Shaner DL, Reider ML. Physiological responses of corn (Zea mays) to Ac 243,997 in combination with valine, leucine, and isoleucine. Pest Biochem Physiol, 1986;25:248-257.

Singh BK. Biosynthesis of valine, leucine e isoleucine. In Singh BK, editor. Plant amino acids. New York: Marcel Dekker,1999. p 227-47.

Wittenbach VA, Abell LM. Inhibition of valine, leucine and isoleucine biosynthesis. In Singh BK, editor. Plant amino acids: Biochemistry and biotechnology. New York: Marcel Dekker, 1999. p 385-416.

Wittenbach VA, Aulabaugh A, Schloss JV. Examples of extraneous site inhibitors and reaction intermediate analogs: acetolactate synthase and ketol-acid reductoisomerase. In Frehse 
$\mathrm{H}$,editor. Proceedings of the seventh international Congress of Pesticide Chemistry (IUPAC). Hamburg, 1991. p. 151-160.

Yoshida S, Tazaki K, Minamikawa T. Occurrence of shikimic and quinic acids in angiosperms. Phytochem 1975;14:195-7.

Zabalza A, Gaston S, Ribas-Carbó M, Orcaray L, Igal M, Royuela M. Nitrogen assimilation studies using ${ }^{15} \mathrm{~N}$ in soybean plants treated with imazethapyr, an inhibitor of branched-chain amino acid biosynthesis. J Agric Food Chem 2006;54:8818-23.

Zabalza A, Gonzalez EM, Arrese-Igor C, Royuela M. Fermentative metabolism is induced by inhibiting different enzymes of the branched-chain amino acid biosynthesis pathway in pea plants. J Agric Food Chem 2005;53:7486-93.

Zabalza A, Orcaray L, Gaston S, Royuela M. Carbohydrate accumulation in leaves of plants treated with the herbicide chlorsulfuron or imazethapyr is due to a decrease in sink strength. J Agric Food Chem 2004;52:7601-06.

Zabalza A, Orcaray L, Igal M, Schauer N, Fernie AR, Geigenberger P, van Dongen JT, Royuela M. Unraveling the role of fermentation in the mode of action of acetolactate synthase inhibitors by metabolic profiling. J Plant Physiol 2011;168:1568-75. 


\section{Legends of Figures}

Fig. 1. Shoot length (A) and root length (B) in control pea plants or plants treated with imazethapyr or 200 and $500 \mu \mathrm{M}$ CPCA supplied to the nutrient solution. Mean \pm SE $(n=8)$. The symbols indicate significant differences between the control and imazethapyr $\left({ }^{\wedge}\right), 200 \mu \mathrm{M} \mathrm{CPCA}\left({ }^{\star}\right)$ or 500 $\mu \mathrm{M}$ CPCA (\#) treatments on the indicated days $(p \leq 0.05)$.

Fig. 2. Shoot $(A)$ and root $(B)$ acetolactate content in control pea plants or plants treated with imazethapyr or $200 \mu \mathrm{M}$ or $500 \mu \mathrm{M}$ CPCA supplied to the nutrient solution. Mean $\pm \mathrm{SE}(n=4)$. The symbols indicate significant differences between the control and imazethapyr $\left({ }^{\wedge}\right), 200 \mu \mathrm{M} \mathrm{CPCA}\left(^{*}\right)$ or $500 \mu \mathrm{M}$ CPCA $(\#)$ treatments on the indicated days $(p \leq 0.05)$.

Fig. 3. Net photosynthesis in control pea plants or plants treated with imazethapyr, $200 \mu \mathrm{M} \mathrm{CPCA}$ or $500 \mu \mathrm{M} \mathrm{CPCA}$ in the nutrient solution. The measurements were performed daily on the youngest fully expanded leaves. Mean \pm SE $(n=6)$. The symbols indicate significant differences between the control and imazethapyr $\left({ }^{\wedge}\right), 200 \mu \mathrm{M}$ CPCA $\left(^{*}\right)$ or $500 \mu \mathrm{M}$ CPCA (\#) treatments on the indicated days $(p \leq 0.05)$.

Fig. 4. The fructose (A), glucose (B), sucrose (C) and starch (D) contents in the leaves of control pea plants or plants treated with imazethapyr, $200 \mu \mathrm{M}$ CPCA or $500 \mu \mathrm{M}$ CPCA. Mean \pm SE $(n=4)$. The symbols indicate significant differences between the control and imazethapyr $(\wedge), 200 \mu \mathrm{M}$ CPCA $\left({ }^{*}\right)$ or $500 \mu \mathrm{M}$ CPCA $(\#)$ treatments on the indicated days $(p \leq 0.05)$.

Fig. 5. The sucrose (A) and starch (B) contents in the roots of control pea plants or plants treated with imazethapyr, 200 or $500 \mu \mathrm{M}$ CPCA supplied to the nutrient solution. Mean \pm SE $(n=4)$. The symbols indicate significant differences between the control and imazethapyr $\left(^{\wedge}\right), 200 \mu \mathrm{M} \mathrm{CPCA}\left(^{*}\right)$ or $500 \mu \mathrm{M}$ CPCA (\#) treatments on the indicated days ( $p \leq 0.05)$.

Fig. 6. Total free amino acid contents in the leaves (A) and roots (D) and the percentages of selected amino acids with respect to the total free amino acid contents in the leaves (B and $C)$ and roots $(E$ and $F$ ) of control pea plants or plants treated with imazethapyr, $200 \mu \mathrm{M}$ CPCA or $500 \mu \mathrm{M}$ CPCA. B and E: branched-chain amino acids; $C$ and F: aromatic amino acids. Mean $\pm S E(n=4)$. 
The symbols indicate significant differences between the control and imazethapyr $(\wedge), 200 \mu \mathrm{M}$ CPCA $\left(^{*}\right)$ or $500 \mu \mathrm{M}$ CPCA $(\#)$ treatments on the indicated days $(p \leq 0.05)$.

Fig. 7. Quinate content in the leaves of control pea plants or plants treated with imazethapyr, 200 or $500 \mu \mathrm{M}$ CPCA supplied to the nutrient solution. Mean \pm SE $(n=4)$. The symbols indicate significant differences between the control and imazethapyr $\left({ }^{\wedge}\right), 200 \mu \mathrm{M} \mathrm{CPCA}\left({ }^{*}\right)$ or $500 \mu \mathrm{M}$ CPCA (\#) treatments on the indicated days $(p \leq 0.05)$. 


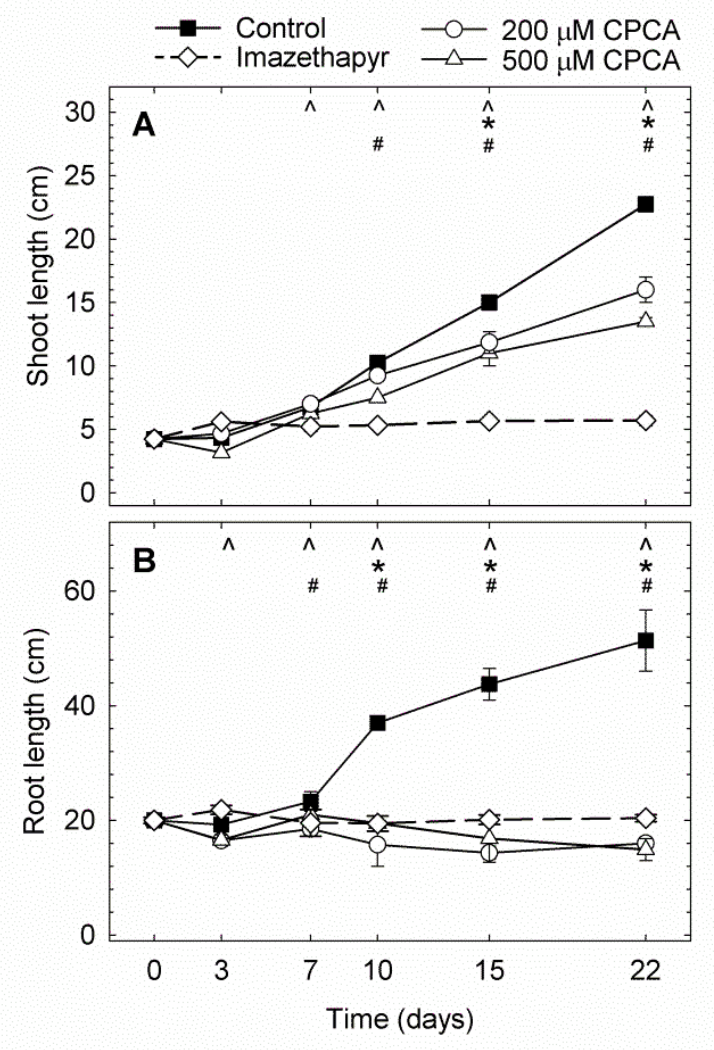

Figure 1.

Zabalza et al. 2012 


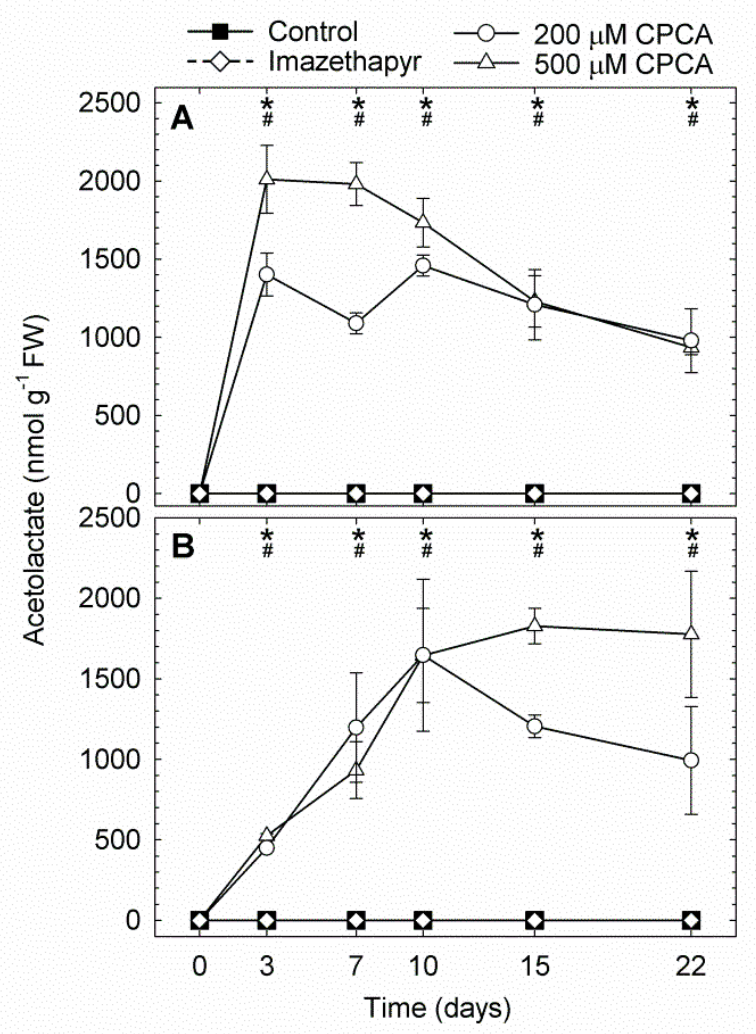

Figure 2.

Zabalza et al. 2012 


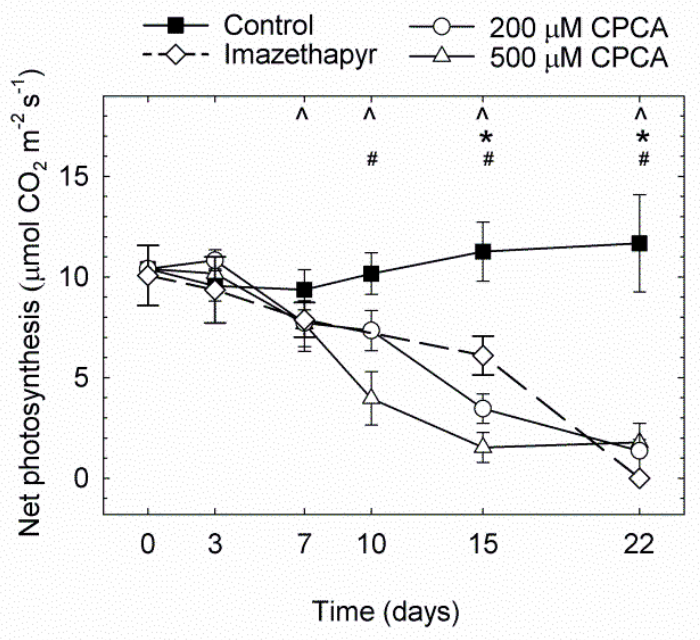

Figure 3.

Zabalza et al. 2012 


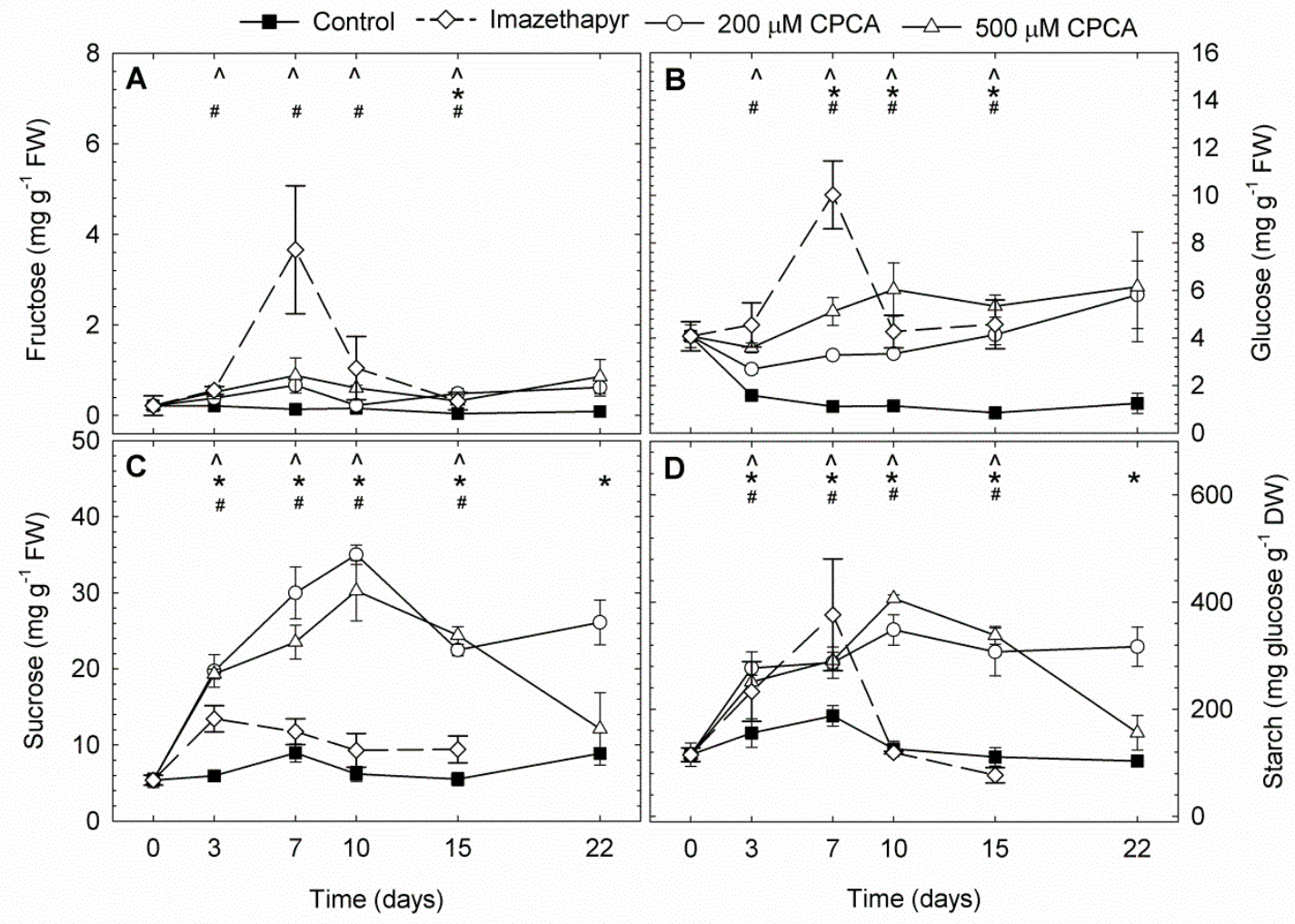

Figure 4.

Zabalza et al. 2012 


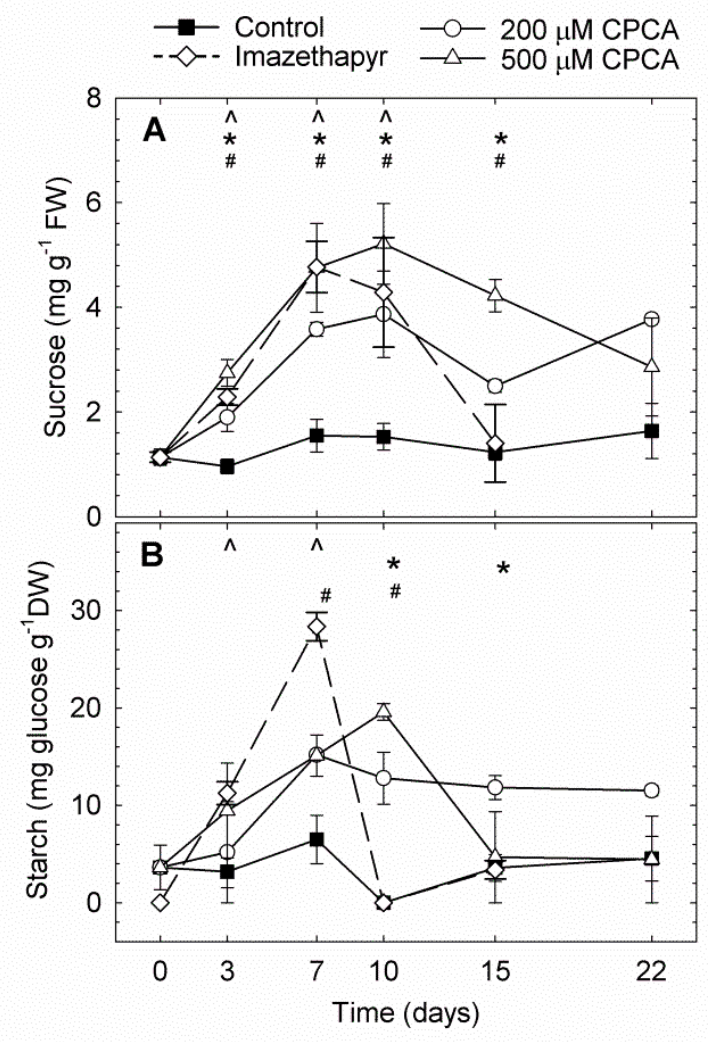

Figure 5.

Zabalza et al. 2012 




Figure 6.

Zabalza et al. 2012 


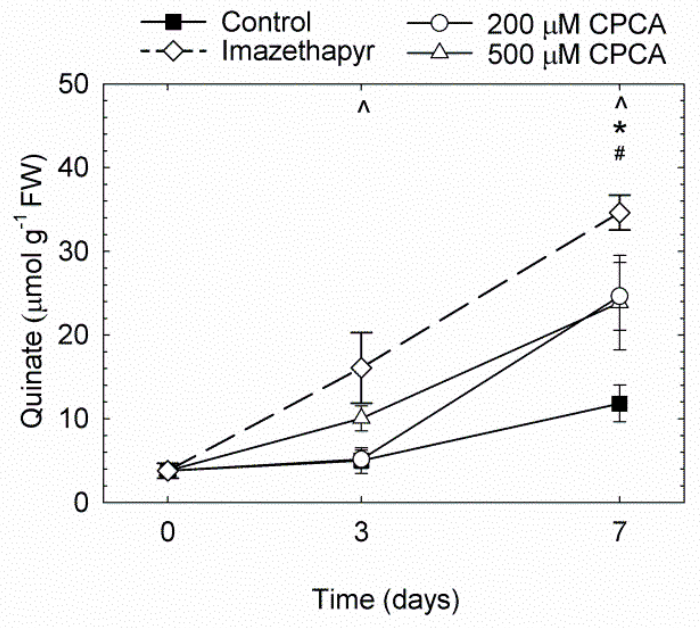

Figure 7.

Zabalza et al. 2012 